\title{
Entrelacs
}

Cinéma et audiovisuel

$8 \mid 2011$

Imaginaire

\section{Chemin faisant... Avec Abbas Kiarostami}

\section{Philippe Ragel}

\section{OpenEdition}

Journals

Édition électronique

URL : http://journals.openedition.org/entrelacs/235

DOI : 10.4000/entrelacs.235

ISSN : 2261-5482

\section{Éditeur}

Éditions Téraèdre

\section{Édition imprimée}

Date de publication : 1 février 2011

ISBN : 978-2-360850-03-7

ISSN : 1266-7188

\section{Référence électronique}

Philippe Ragel, « Chemin faisant... Avec Abbas Kiarostami», Entrelacs [En ligne], 8 | 2011, mis en ligne

le 01 août 2012, consulté le 19 avril 2019. URL : http://journals.openedition.org/entrelacs/235 ; DOI :

10.4000 /entrelacs.235

Ce document a été généré automatiquement le 19 avril 2019.

Tous droits réservés 


\title{
Chemin faisant... Avec Abbas Kiarostami
}

\author{
Philippe Ragel
}

Chemin faisant... ${ }^{1}$ Riches en détours, sinueux, parfois emboîtés, ouverts sur le paysage et les êtres qui le traversent plutôt que centrés sur le récit, ne laissant rien à la vanité du définitif fermé sur sa clôture, les films du cinéaste iranien Abbas Kiarostami sont comme une invitation toujours renouvelée à explorer d'immenses territoires imaginaires. Mais de quel imaginaire voulons-nous parler ici? Traiter d' «imaginaire» en pays persan, suppose en effet qu'on le fasse avec prudence, qu'on entoure aussitôt le terme de quelques guillemets tant les travaux d'Henry Corbin en la matière lui ont donné le sens que l'on sait. Ainsi lui préférer, «parce que ce mot, dans l'équivoque courante, préjuge de la réalité atteinte ou à atteindre $~^{2}$, celui de mundus imaginalis, ou monde imaginal, plus enclin à transcrire l'expérience mystique avec laquelle se confond, selon le philosophe, l'imaginaire persan tout empreint de culture shiite. De cet héritage immense qui depuis plus d'un millénaire féconde et pénètre toute la culture persane jusqu'à déborder d'ailleurs les limites du religieux, l'œuvre d'Abbas Kiarostami que le poétique travaille avec une intensité sans égale, ne saurait évidemment se départir. Quand même d'une complexité infinie, à cet unique dialogue avec le monde suprasensible gardons-nous de la ramener trop vite. Le régime propre de l'imaginaire, déhiscent, abstrus, en effet n'y résisterait pas longtemps, non plus le cinéma et les très nombreuses autres influences qui par ailleurs nourrissent 1 'œuvre du cinéaste.

2 Pour le dire rapidement, outre la poésie (de Rûmi à Sohrab Sepehri, d'Omar Khayyâm à Forough Farrokhzad), héritage zoroastrien, références à l'art de la miniature et symbolique cosmogonique composent en effet un paysage d'arrière plan qui toujours semble parler à voix basse dans l'œuvre du cinéaste. Quelque chose d'une grande ouverture sur l'imaginaire la traverse, ou plutôt l'habite tant rien ne s'impose ici en une franche direction d'orgueil trop rempli et de certitudes acquises. Les films de Kiarostami habitent ainsi un monde qui en retour les nourrit; ils ne prennent pas des vues mais rendent à la vue, car le geste de prendre tout en donnant et en se donnant, par respect se 
retire. Il y a dans ce donner qui s'absente un art du retrait et du vide propre au monde oriental, « cette apparition du lointain dans son retrait » dont parle Youssef Ishaghpour à propos des photographies de Kiarostami ${ }^{3}$, un art du chuchoté, du murmure ou du souffle aussi comme manifestation divine, mais encore un art de ce qui se couche, ou veut se coucher, au lieu de se dresser (Le goût de la cerise) et auquel les travaux de Gilbert Durand ont su reconnaître une grande puissance d'ouverture sur l'imaginaire. Les nombreux motifs comme les différents sujets développés dans le cinéma de Kiarostami prolongent et fécondent sans conteste cet art du repli ouvert sur une imagination active, mais aucun sans doute ne l'affirme aussi vivement que ce motif du chemin dont nous voulons ici vous entretenir et qui en constituerait comme une espèce de guide.

3 Motif de reconnaissance du cinéma de Kiarostami depuis au moins Où est la maison de mon ami ?, le chemin agit telle une source, tel un fluide paysager doué d'une grande force d'élancement en même temps qu'il invite à la traversée contemplative. Son devenir comme son origine jamais ne s'épuise car, sans début ni fin, toujours d'un ailleurs le chemin surgit et dans le lointain s'enfonce et se poursuit. S'il nous parle de cette absence où il se retire comme de ce devenir où il attire, le chemin est aussi passage, conduite provisoire, inconstante, éphémère, voie particulière parmi la somme des possibles pour les personnages qui l'empruntent et pour le regard du cinéaste qui à sa vue choisit de nous attacher. Sa ligne d'abord brisée, ses virages angulaires dont la courbe ensuite se précise (à partir du Goût de la cerise), suggèrent un rapport poétique au monde dont le régime narratif des films de Kiarostami semble hériter par la forme caminale qu'ils adoptent. Nous avons ailleurs observé la puissance subversive de cette composition inaugurale en forme de $\mathrm{Z}$ retourné profitant à la course du jeune Ahmad lancé sur le chemin de Poshté, suppléée par la présence de l'arbre au faîte de la colline. ${ }^{4}$ Nous avons évoqué aussi, toujours associée à la figure de l'arbre (l'olivier), sa fonction itérative dans la trilogie de Koker. En revanche, nous avons peu développé le changement de régime de genres que le motif du chemin introduit parfois, comme la bifurcation structurelle qu'il imprime à la narration dès que sa puissance figurale se manifeste, non plus l'évolution graphique et de traitement qu'il devait subir entre la trilogie de Koker et, par exemple, le très récent essai filmique Roads of Kiarostami, variations caminales riches en échappées imaginaires que les lignes suivantes, pas à pas, se proposent de suivre.

Emprunter le chemin signifie souvent chez Kiarostami franchir un seuil, oser une traversée pour un autre monde, passer à d'autres modalités d'être au monde. Agissant comme un virage narratif, le chemin libère des modulations sonores et visuelles inédites aussitôt plus suggestives. Lorsqu'Ahmad franchit par exemple la colline où court le célèbre sentier, Où est la maison de mon ami? qui adoptait jusque-là un style réaliste, plonge en effet dans un étrange conte fantastique. Passé le chemin, l'enfant se heurte au cauchemar de l'indiscernable; l'environnement devient hostile et rebelle à son entreprise : miaulements, voix diffuses, pierres jetées hors-champ, silhouettes fugaces, personnages masqués (le porteur de fagot, le fils d'Ali Hemati caché par le volet). Dans Où est la maison de mon ami ?, passer le chemin revient à passer de l'autre côté. Les contours perdent de leur précision, le monde des certitudes et des bonnes résolutions (trouver Nématzadeh qui habite Poshté), le cède à celui de l'indétermination et de l'incertitude. Chaque épreuve rencontrée n'est dépassée que pour être aussitôt niée comme si ce monde se jouait de l'enfant, se retirait à mesure qu'Ahmad y pénétrait. Sitôt franchi le sentier qui mène à Poshté, Ahmad évolue dans un univers empli de mystères et voué à la dissémination. Les noms ne veulent plus rien dire et à l'infini se multiplient, la 
géographie tremble, l'espace se dilate. Ainsi le village de Poshté qui n'est pas un village, apprend l'enfant dès son arrivée dans ce monde étrange dont deux femmes $\mathrm{p}$ ostées en vigie sur un balcon semblent garder l'entrée. Dans cet univers qui n'est pas géocentré, qui n'a pas de centre mais se divise et se ramifie en plusieurs hameaux, habiter Poshté ne veut rien dire, comme est-il tout aussi vain de rechercher un certain Nématzadeh au pantalon marron parmi la foule de Nématzadeh au pantalon sans doute marron qui peuple les environs. L'innocence propre au monde de l'enfance constitue dès lors la plus belle promesse d'imaginaire contre la raison que lui oppose, dans le film, la parole des adultes. Ahmad balbutie, son enquête piétine et tourne littéralement en rond, en boucle, de Koker à Poshté et inversement, cinq fois, ${ }^{5}$ préfigurant ce monde clos sur lui-même où, selon la force centripète que produit en retour toute révolution centrifuge, le film à la fin le ramènera. Nous voulons dire en son for intérieur que métaphorise si bien la chambre où l'enfant, enfin en paix avec lui-même, pénètre son double Nématzadeh en s'y substituant. Alors que se manifestent les forces magiques de l'univers quand l'orage gronde, Ahmad fait les devoirs de Nématzadeh littéralement à sa place, et trouve, ainsi, la maison de l'ami.

5 C'est la rencontre avec le vieux menuisier, alors que la nuit est tombée, qui finira de précipiter le film dans l'atmosphère du merveilleux que préparent depuis la fin de la classe les errances d'Ahmad autour de l'introuvable maison de l'ami. Et là encore le chemin que les obscures ruelles dessinent nuitamment, scelle cet ultime changement de registre. A l'instar d'un guide convoquant pour son commentaire les moucharabiehs, ces fenêtres ajourées dont la particularité est, chose importante dans ce contexte, de voir sans être vu, le vieil homme nous fait pénétrer dans l'abstraction, dans la magie du conte au cœur des Mille et une nuits. Reflets diamantés, découpes de lumière, éclairage rasant sur les murs où dansent des ombres menaçantes, tout concourt à nous plonger dans la feérie de la fable. La nuit favorise l'apparition du double et drape la réalité d'apparence. A la lenteur du vieillard qui maintenant freine le récit, s'ajuste le nouveau mode de progression adopté, ruinant en quelque sorte et définitivement le modèle téléologique qui jusque-là semblait dominer la course d'Ahmad. La déambulation nocturne dame le pion à la dynamique physique du sentier diurne et impose un autre rapport au monde, riche en ralentissements et en pauses : rien ne sert de courir, il faut savoir s'arrêter et écouter ce que le monde suggère implicitement. Dans ce monde où l'obscurité le dispute à la magie des projec tions, où les extrémités scellent d'efficientes alliances comme il en est de la sage vieillesse accordée ici à l'intrépide enfance; dans ce monde où il faut résister au règne du dur, de la froideur minérale qui menace de gagner sur le chaud, le tendre, le végétal des fenêtres de bois d'antan ainsi que s'en inquiète le vieux menuisier, le chemin dessine, non une solution, mais un imaginaire du possible. Il donne accès au symbolique dont on sait la force toujours ouvrante, particulièrement dans la pensée iranienne où « le symbole annonce un autre plan de conscience que l'évidence rationnelle; il est le 'chiffre' d'un mystère, le seul moyen de dire ce qui ne peut être appréhendé autrement $»^{6}$, mystère que la poésie dévoile ici avec avantage, « à deux pas de la fleur [...] / Au pied de la fontaine où jaillissent les mythes de la terre ", ainsi qu'y invite le poème de Sohrab Sepehri qui inspira au cinéaste le scénario de ce film.

6 Il n'est guère de films de Kiarostami où le passage du chemin n'entraîne de grandes modifications pour le personnage et la mission qu'il s'est donné. A son contact, le chemineau s'affranchit car celui-ci lui dévoile des mystères insoupçonnés et lui révèle le sens de sa quête en en modifiant les modes d'accès. En ce sens, le chemin se présente 
souvent comme une figure libératoire, douée d'une puissance dynamique jamai $\mathrm{s}$ entamée, une figure de l'écart, de la bifurcation, partant de l'insubordination.

Comme l'évoque si bien son jeu vocal (il marmonne, bredouille), le propre d'Ahmad dans Où est ma maison de mon ami ? est de répéter. Dès la deuxième séquence, l'enfant est pris dans la série des répétitions, de la reprise. Et il lui faudra répéter sans cesse, répéter des trajets, obstinément répéter qu'il doit porter le cahier à Nématzadeh. L'expérience d'Ahmad consiste ainsi en un apprentissage du discernement dans ce qui est de l'ordre du répété et du même. La répétition, il faut l'affronter, s'en affranchir pour devenir un sujet libre. Et ce qui permet de s'extraire de l'ordinaire de la répétition, c'est désobéir. Pour accéder à la liberté, il faut transgresser l'ordre de la loi car la liberté est au-dessus, et même hors de la loi sociale ; il faut saisir l'occasion qui se présente, prendre des risques, passer outre les interdictions, oser des rencontres inattendues, bizarres, se mettre en danger, aller en territoire inconnu. C'est bien là ce qu'indique ce chemin en forme de Z retourné qui fait signe dans le paysage. Sa forte iconicité angulaire assigne de brusques changements de régimes et de direction. En ce sens, le chemin de Où est la maison de mon $a m i$ ? est un peu à l'image de ce que Pierre Boutang remarquait dans ses notes de préparation à l'Abécédaire de Gilles Deleuze à propos de la la lettre « $\mathrm{Z} »$ : un signe de la bifurcation que l'on trouve jusque dans les noms des grands philosophes de la révolte, de l'écart, de Spinoza à Nietzsche en passant par Leibnitz ${ }^{7}$. Au modèle aristotélicien des péripéties et téléologique du récit, aux constructions monolithiques du parangon hollywoodien dominant, ce signe-lettre oppose aussitôt une écriture du risque, de l'indéterminé, de la rupture, dans laquelle s'inscrivent pleinement les films de Kiarostami riches en grands moments de bifurcation. A ce titre, comment ne pas évoquer la magistrale clausule du grand embouteillage de Et la vie continue... lorsque, contre toute attente, excédé le père décide de se risquer sur ce chemin de traverse, cette route de fortune qui zèbre la colline et fissure littéralement le plan par la diagonale. A l'itinéraire trop bien tracé de la route principale en contrebas, au flux général et aux comportements grégaires, au scénario convenu (rejoindre Koker par la route tout indiquée et retrouver les deux enfants), cette bifurcation inattendue oppose une autre voie. Laquelle consiste à emboîter justement le pas de l'incertitude, à adopter le régime de l'aléatoire qui résonne jusque dans la construction du récit, en règle générale sinueuse, ou dans son manque de lisibilité apparent et dans l'emploi de certaines figures obtuses et rétentrices comme l'ellipse et la fausse-pis te. Arrêtons-nous un instant sur l'emploi de ces dernières dans Le Vent nous emportera, autre film où la conduite du récit semble particulièrement leur obéir.

Grand film sur la bifurcation s'il en est, Le Vent nous emportera refuse toute forme d'efficacité ou de rentabilité dramatique, à l'inverse de son personnage principal qui est un homme de télévision élevé dans une culture de la performance et de la productivité des images. Construite sur le principe d'une structure plus verticale qu'horizontale à l'image des terrasses qui composent le village et des hauteurs du cimetière où se rend régulièrement Behzad pour recevoir les communications sur son téléphone mobile, ce film recourt fréquemment à l'ellipse, figure équivalent à un « trou » narratif qui travaille le film par l'en-dessous, dans sa diégèse souterraine. Exactement ce qu'évoque ici la fosse que creuse ce terrassier au cimetière et dont on ne verra jamais le visage, précision importante en la circonsta nce car si l'on comprend bien qu'il creuse un trou, on ne saura jamais exactement pourquoi il s'adonne à cette tâche. Cette fosse où l'ellipse trouve son prolongement figuratif, nous apprendrons par la suite qu'elle communique, par le jeu de la métaphore amoureuse, avec cette autre cavité du film qu'est l'obscure étable où Behzad 
ira se procurer un peu de lait. De ces trous plongés dans une obscurité que leur dispute, à l'étable tout au moins, la douce lumière d'une lampe à pétrole, le film tire pourtant toute sa force car s'y manifeste un rapport au monde de nature poétique. C'est par exemple dans cet antre obscur que Behzad récitera le poème de Forough Farrokhzad qui inspira, là encore, le titre du film. Par un effet de retournement saisissant, la figure elliptique qui $a$ priori embarrasse et dissimule (comme le métaphorise ici le trou associé à l'obscurité), tout au contraire libère les puissances suggestives qui sont le propre de la poésie: l'ellipse, loin de réduire en nuisant à la bonne compréhension du récit, accroît parce qu'elle évoque plus qu'elle ne dit, et partant à l'imaginaire profite.

9 Autre figure stimulante, plus malicieuse et très hitchcockienne : la fausse-piste. Celle par exemple, toujours dans Le vent nous emportera, de la chasse au trésor derrière laquelle Behzad cache son projet de reportage pour ne pas attirer l'attention des gens du village. Cette piste donnée dès le début comme fausse s'avère pourtant, par le jeu métaphorique, être la bonne. Car Behzad finira bien par trouver un trésor, cependant pas tout à fait celui qu'il était venu chercher dans-la-mort (le reportage sur la cérémonie de deuil et le rituel de scarifications qui lui est associé), mais celui inestimable qu'est la vie et dont le médecin lui ouvre les portes sur les vers chantés d'Omar Khayyam lors de la somptueuse séquence où ils serpentent à motocyclette à travers les chemins coupant les blés.

Ellipse, fausse-piste, autant de figures qui ne facilitent pas la lisibilité, qui réclament une souplesse intellectuelle et obligent à de nombreuses allées et venues entre ce que la continuité dévoile progressivement et ce que l'on croit en être de l'histoire à l'instant même de ce dévoilement. D'aucuns diront que ces procédés scénaristiques en chicane n'ont rien de bien novateur, Hollywood en ayant par exemple produit un grand nombre (le cas emblématique d

11 rquote Hitchcock, encore). A la différence près que ceux-ci débouchent toujours, ou presque, sur une résolution finale cohésive qui, peu ou prou, vise à définitivement figer l'histoire dans le marbre. Chez Kiarostami, en revanche, et à l'image des fins ouvertes de ses films, rien ne permet le plus souvent d'affirmer ce qu'il advient des personnages et ce qu'il en est des objectifs qu'ils s'étaient donné d'atteindre.

Ces procédés narratifs en disent long sur les intentions de l'auteur et sur sa vision du cinéma comme instrument et acte de pensée. A l'instar du graphisme des chemins et par l'entremise de ces figures narratives rétentrices, le récit n'adopte une construction caminale que pour mieux ouvrir le champ herméneutique contre le sens unique que la continuité impose, au cinéma, trop souvent de facto. Dans cette approche qui nous contraint à construire la logique dramatique des faits à partir d'éléments dispersés, de non-dits, d'impératifs différés, réside une idée chère au cinéaste iranien qui ne consiste pas tant à gêner notre lecture qu'à se retirer le plus possible du pouvoir que détient l'œuvre d'art de nous soumettre à l'autorité de son créateur. Cette question de la liberté au cœur même du cinéma de Kiarostami croise d'ailleurs celle-là même que féconde de manière très productive la part que le cinéaste réserve fréquemment à la mise en attente d'événements programmés par la diégèse et qui tardent, voire jamais ne parviennent à se manifester. Ainsi l'objectif qui est de retrouver les deux enfants dans Et la vie continue..., ainsi le suicide de Monsieur Badii dans Le goût de la cerise, ou encore, dans Le vent nous emportera, la mort de cette vieille femme longtemps différée. Car, pour Kiarostami, le cinéma n'atteint son degré d'intense vitalité que lorsque impuissants à rétablir l'ordre d'un scénario inscrit même en filigrane, ses personnages s'abandonnent à l'imprévu, aux rencontres inattendues et aux expériences provisoires dont nous pourrions croire qu'un 
monde les sépare des objectifs qu'en apparence le cinéaste leur a fixés. Là où le modèle dominant veut imposer la prise en charge totale du spectateur (au singulier naturellement...) jusqu'à neutraliser ses émotions par une surenchère dramatique, le cinéma de Kiarostami cultive tout au contraire temps morts, lenteur, débrayage, attente, retardements, syncopes. Cette sinuosit_e9 du récit que le motif du chemin en lacet figure sans peine, ce sont, outre les figures narratives évoquées plus haut, toutes ces absences, ces énigmes, ces silences que l'imaginaire du spectateur particulièrement mis à contribution est convié à féconder. Ce qu'annonce, pour peu qu'on s'y arrête un instant, l'admirable ouverture du Vent nous emportera avec son paysage tout en rondeurs et arabesques, sa route dont le balancement dans les collines illustre déjà magnifiquement ce régime sinueux de l'incertitude, cette architecture des impératifs différés, cette logique du retardement et de la bifurcation que l'on retrouve, comme nous l'avons vu, dans la structure même du récit.

Cette séquence d'ouverture dans laquelle Charles Tesson voit «le premier signe d'un dysfonctionnement profond qui va perdurer $"^{8}$ offre un bon exemple de cette " évidence " (Jean-Luc Nancy) dont joue le cinéma de Kiarostami. Cet incipit s'ouvre, au sens le plus fort, sur un paysage mordoré dont la beauté plastique tout en camaïeu d'ocres et délicates courbures, emporte d'emblée notre totale adhésion. Au loin, en contrebas, un $4 \times 4$ descend la route en lacet qui serpente à travers la colline. Nous sommes en territoire ami, reconnaissant là une posture chère à l'opus précédent, Le goût de la cerise, premier signe de reconnaissance qui reconduit le procédé d'emboîtement propre à la démarche que poursuit le cinéaste de film en film. Tout va bien semble-t-il, mais cependant quelque chose dérange. Des sons nous parviennent, nous identifions des voix, notre écoute se tend et rapidement se positionne. Nous sommes en un point d'écoute subjectif qui nous situe étrangement dans l'intimité de la voiture, avec ses occupants, emplacement dont l'image pourtant nous exclut par l'objectivité d'un point de vue lointain et dominant la situation en plan général. Ainsi, le son donne aux personnages une présence que l'image leur refuse obstinément.

Dès l'ouverture, on n'entend donc pas d'où l'on regarde et on ne voit pas d'où l'on écoute. Ce que confirme, aussitôt après, le débat autour de l'arbre-repère que voient les occupants de la voiture alors que nous ne jouissons pas encore de sa présence dans le champ, et qu'un panoramique nous dévoile au moment même où les occupants disent qu'il échappe maintenant à leur vision car la colline fait alors obstacle. Ce régime contradictoire adopté par l'image et le son, et qui fait dire à Charles Tesson dans sa très belle analyse que "pour le spectateur, passager-témoin en porte-à-faux, la présence aux choses, à l'arbre isolé, ne se fait pas sur le même mode... ni au même rythme que celle des personnages $"{ }^{9}$, notons néanmoins combien l'homogénéisent la route tout en méandres et courbures, les collines tout en rondes cambrures, le trajet de la voiture aux fluides entrées et sorties de champ, ou encore l'atmosphère cuivrée du paysage rehaussée de verts intenses.

De n'avoir cédé ni à l'un ni à l'autre de la confusion ou de l'absolue suture, ce maillage subtil du son et de l'image tire toute sa force: le montage ne contracte pas l'espacetemps, comme on pourrait le croire si l'eurythmie à laquelle profite le contrepoint visuel ne faisait ici son ouvrage; il le dilate, l'oxygène, le porte à la mesure de cette beauté d'autant plus vaste que l'origine du son nous est justement refusée, que l'image paradoxalement la repousse au loin et l'absente. Nous ne roulons pas «sans but » ainsi que le craint Behzad, le conducteur encore masqué de cet étrange voyage. À l'attention de 
ceux qui se croyaient perdus, sans repères, Kiarostami ouvre une brèche sur l'immobile foyer que tout mouvement gouverne. De ce cinéma des extérieurs, on ne dira jamais assez tout ce qu'il a de tellurique et de solaire mêlés, de prompt à s'engager et à nous engager. À l'adresse d'Ali qui dans la voiture affirme qu' «il n'y a rien près de l'arbre », Behzad répond qu'il se trompe, "qu'il y a près de l'arbre une ruelle boisée plus verte que le songe de Dieu », citant un vers du poème de Sohrab Sepehri à l'origine, faut-il le rappeler, de Où est la maison de mon ami?

Comme un appel, l'arbre oriente notre regard. Il lui indique « une terre visionnaire » où Mojdeh Famili voit la réminiscence «du jardin-paradis qui apparaît avec la cosmologie Mazdéenne, la religion de Zarathoustra $»^{10}$, bien avant que l'Islam ne recycle cet archétype. Aux confins du chemin qui est un itinéraire d'existence et que le film dessinera implicitement, existe un territoire inconnu, encore vierge, un territoire imaginaire comme un champ élargi à l'écoute et au voir qui est aussi une pensée du cinéma. Nous voulons parler de cet « approfondissement de la chose visible selon son sens obscur et en quelque sorte imminent $»^{11}$, lieu poétique qu'habite de manière précise, attentive, le cinéma de Kiarostami et que Behzad, sur le mode de la plaisanterie en ce début de film, n'est pas encore à même de soupçonner. Siah Dareh, la " Vallée noire » vers laquelle il se dirige avec ses certitudes d'ingénieur et son programme de reportage, se chargera de lui en murmurer le mystère, ou, comme y invite une fois encore le titre, de lui en souffler le secret.

Comme nous l'évoquions en préambule, nous voudrions fermer ces quelques pages en revenant sur l'évolution graphique du motif du chemin que nous avons pu observer entre la composition inaugurale de où est la maison de mon ami ? et, par exemple, le très récent essai photo-filmique Roads of Kiarostami. Nous notions tout à l'heure ses caractéristiques plastiques angulaires particulièrement accordées, nous semble-t-il, à la portée subversive de la décision de l'enfant et aux brusques changements de régime de genre observés ensuite dans Où est la maison de mon ami ? Saillants, aigus, les virages du chemin y ont l'aspérité et la rugosité de la révolte tenace. Ils ne tournent pas, n'enrobent pas, n'enveloppent pas, mais cassent, brisent, tailladent la colline, en ébrèchent les contours. Oublions pour l'heure la question de la répétition du motif au sein de la trilogie pour observer combien cette composition subit une singulière évolution dès l'opus suivant, non dans la « reprise $»^{12}$ effective de ce plan (cité environ au milieu du film), mais dans ce qui, lors du grand final, en constituerait comme son pendant, ou son élargissement. Rappelons-nous comment Farad, après une dernière "panne » et un dernier regard en direction de la colline voisine où, sur la crête, les silhouettes de deux enfants se découpent (peut-être ceux qu'ils recherchent depuis le début de son voyage); comment donc Farad remonte à bord de sa Renault 5 jaune pour tenter l'ultime ascension de la route qui traverse la montagne le séparant de Koker, destination laissée pour hypothétique. Comment, en un long plan-séquence en pano-travelling arrière optique, peu à peu l'échelle de plan (rapprochée sur la voiture) se desserre au profit du paysage de montagne que zèbre la route et que la voiture peine à gravir (plan général). Outre les effets de relance de régime de narration que suggèrent avantageusement les ratés et redémarrages de la voiture suppléés par le changement du thème musical (Vivaldi) et par la réconciliation observée du pédestre et du motorisé (finalement Farad fait monter l'homme à pied qui l'a d'abord aidé en poussant la voiture), il est ici tout à fait intéressant de noter combien la composition graphique que dessine la route à flanc de montagne, évolue. Au cours de cette action qu'accompagne le double mouvement d'appareil, en effet 
le cadre délaisse progressivement le jambage inférieur que dessine la route en bas pour celui, supérieur, que l'échelle de plan et l'axe initiaux ne permettaient pas de voir. Ainsi, perç ue tout d'abord en forme de $\mathrm{Z}$, la composition graphique de se retourner au fur et à mesure du panoramique horizontal qui double le changement de focale et de retrouver la forme inaugurale de Où est la maison de mon ami ? Sauf que, nous semble-t-il, quelque chose a singulièrement changé. Non seulement les moyens filmiques d'accéder à sa vision (plan fixe versus mouvement d'appareil), mais surtout la caractéristique graphique des virages qui la composent. Sans être complètement courbes, en effet ceux-ci n'ont plus l'aspérité de la composition inaugurale de la trilogie de Koker. Au glissement du cadre, sa douce lenteur, semblent ici répondre ces angles qui se sont arrondis et promettent non tant une fin heureuse à l'entreprise que la possibilité d'un monde, un monde pleinement accordé à la vie qui, bien au-delà de ce film qui s'achève, toujours continue, se restaure malgré la mort et les cicatrices que laisse la catastrophe : « quand nous considérons les montagnes, il y a toujours en nous, plus ou moins forte, plus ou moins consciente aussi, l'idée du col, du passage, l'attrait de ce qu'on n'a pas vu $»^{13}$.

La sinuosité qu'installe ce final trouvera à s'accomplir dans les œuvres postérieures où le chemin comme trace visible pourra aller jusqu'à s'effacer par exemple dans le final d'Au travers des oliviers, emporté par un bruissement du corps sur le végétal, par ce que Jean Mottet décrit comme un "glissement continu de la minuscule silhouette qui mobilise autour d'elle des forces liantes, à l'instar de l'écorchure du sentier, désormais dissoute dans l'étendue herbeuse ${ }^{14}$. De la douceur enveloppante de la boucle caminale, on ne saurait néanmoins trop se méfier. Son attraction se révèle parfois d'une beauté crépusculaire. Dans Le goût de la cerise, la courbe chorégraphie que dessinent les chemins en lacet dans l'atmosphère de cet automne cuivré pleinement accordée à l'affliction qui ronge Monsieur Badii, n'instaure rien d'autre que l'ivresse morbide d'une danse macabre. Quelque chose du chemin s'est assoupli depuis Ou est la maison de mon ami ?, mais dans sa compulsive attirance pour l'ivresse mortifère qu'il suggère aussi. La ronde des chemins agit telle une spirale hypnotique. Monsieur Badii ne cherche pas quelqu'un pour l'aider à accomplir sa tâche comme tout le laisse croire, il tourne en rond, envoûté, ensorcelé par cette valse enivrante autour d'un point nodal qui l'épouvante et le fascine tout à la fois, le point noir de sa mort physique et le trou (la tombe) qui l'accompagne. A cette chute existentielle répondra, ainsi que nous l'avons évoqué, l'ouverture libératoire du film suivant, Le vent nous emportera, et son sujet, filmer une cérémonie de deuil à laquelle Behzad devra apprendre à renoncer. Que cet incipit imprime au motif du chemin une puissance d'ouverture poétique d'une profondeur inestimable, nous ne saurions trop le rappeler, mais aucun ne l'élève aussi puissamment que l'essai photo-filmique Roads of Kiarostami sur lequel nous voudrions conclure

Ce film commandé à Kiarostami en 2005 pour le soixantième anniversaire de l'explosion de la première bombe atomique à Hiroshima consiste en quatre photomontages réunis par thèmes entrecoupés de trois films où nous voyons le cinéaste sillonner les routes de montagne à bord de son 4X4 (le dernier, un peu différent, le montre à pied d'œuvre), tandis que nous l'entendons évoquer sa fascination (sic) pour la nature et les chemins. Audelà du dispositif qui, tout au moins dans les deux premiers petits films, rappelle étrangement l'ouverture du Vent nous emportera, ce qu'il faut remarquer réside, nous semble-t-il, dans la manière dont le cinéaste a conçu les parties consacrées au montage de ses photographies de paysage. Et plus particulièrement ce dialogue qu'il instaure entre les images fixes et le cinéma qui les met en mouvement. Et comment cette mise en 
mouvement se fait justement au rythme du chemin, blanc sur le sombre des champs dans les deux premières séries photomontées, noir sur fond de neige dans la troisième. Sur une musique de Carl Maria von Weber dont la facture romantique sied à l'entreprise, l'agencement des chemins dont s'empare la caméra, règle un véritable cheminement intérieur. La sinuosité qui nous a beaucoup occupé tout au long de ces pages atteint ici un point d'acmé inégalé. Un mouvement ininterrompu, d'u ne douceur extrême, embrasse ce flux et reflux du plus petit au plus grand quand la caméra pénètre à l'intérieur des photographies ou alors s'en retire. Le balancement que nous évoquions à propos du chemin dans l'ouverture du Vent nous emportera trouve ici son plein aboutissement. Car les compositions caminales de Kiarostami écrivent littéralement le monde en poésie, y dessine des lettres dans une langue qui entretient une analogie évidente avec le farsi. Il y a chez Kiarostami une véritable calligraphie du paysage dans son art de mettre en scène le chemin. Ses ordonnancements courbes et allongés sont de cette langue fluide écrite au calame dans un style nasta'liq étiré, élégant et léger. Ils ont du glyphe persan ses contrastes d'épaisseur, tout en pleins et déliés. Ici le renflement d'un contour, là le galbe d'un arrondi, la finesse d'un étirement plein d'élancement chargé que l'angle adopté pour photographier ou filmer le chemin, creuse ou alors tempère. Et le mouvement compte dans ces phrases qui semblent de ce chemin, là-bas, nous être soufflées. La camérapinceau de Kiarostami d'une sensualité attentive, caresse, glisse, entre et se retire. La danse des chemins, leur ligne orbiculaire, imprime son mouvement à la caméra comme envoûtée par l'onde produite. Le cinéma redessine ces trajectoires en noir et blanc frappées d'irréalité comme un guide ouvrant sur des territoires imaginaires où la pensée devient « fluente ». Le monde en suspension photographique renaît par le mouvement. De ces contournements, de ces ondulations et méandres, de ces recadrages vagabonds où se précipitent les fondus enchaînés qui lentement effacent une image dans l'apparition de la suivante, l'œil s'abreuve. A leur rythme, la fixité de l'instant reprend vie comme un cœur qui se mettrait à battre. Le regard du ciné aste particulièrement soucieux du mouvement de ces traces, montre comment ces images s'ouvrent et comment nous rentrons dedans, comment quelque chose d'indicible monte du centre et sourd inépuisablement $\mathrm{du}$ fond. C'est une chose qui rend sensible une distance, qui jalonne une étendue, à la fois à l'arrêt et flottante. La caméra nous prend par les yeux pour un voyage au pays des variations courbes et instables. Nous allons et venons, virons et chaloupons au gré de cette conduite rassurante mais qui ne retire rien à l'incertitude de cet arrière fond d'où le chemin surgit, rien à l'énigme de cet horizon où il se retire. Car le chemin, c'est aussi cela, ce qui saisit en s'usant, en disparaissant, vie et mort jusqu'au bout de la route toujours mêlées, tressées, comme cette photo finale du chien qui s'enflamme sur fond $\mathrm{d}$ uote explosion atomique dans l'observation assumée et consumée de ses cendres.

\section{NOTES}

1. Comme un hommage à Jacques Lacarrière et son récit autobiographique du même titre.

2. L'imagination créatrice dans le soufisme d'Ibn'Arabi, Ed. Médicis-Entrelacs, Paris, 2006, p. 26.

3. Le réel, face et pile, Farrago, Tours, 2000, p. 15. 
4. « L'olivier du Gîlan. Résurgences... ». L’Arbre, Entrelacs n 6, dir. Philippe Ragel, Toulouse, mars 2007, pp. 103-118.

5. Dans Et la vie continue..., puis Au travers des oliviers, reviendra cette symbolique du chiffre " cinq ".

6. Henry Corbin, op. cit., pp. 35-36.

7. «L'Abécédaire : Gilles Deleuze à la lettre », Vertigo n² 25, printemps 2004, pp. 81-95, mais plus particulièrement page 95 pour la reproduction de la note en question.

8. « Le secret magnifique », Cahiers du cinéma, n 541, déc. 1999, p. 27.

9. Ibid., p. 27.

10. «Le jardin du paradis dans la miniature persane et l'arbre dans le cinéma de Kiarostami ", L'arbre dans le paysage, dir. Jean Mottet, éd. Champ Vallon, coll. « Pays / Paysages », Seyssel, 2002, p. 157.

11. Philippe Jaccottet, Paysages avec figures absentes, Gallimard Nrf, coll. «Poésie », Paris, 1976, p. 46.

12. Il ne s'agit pas en effet d'une reprise littérale de ce plan, mais de sa recomposition actualisée.

13. Promenade sous les arbres, La Bibliothèque des Arts, Paris, p. 63.

14. «Du cheminement à l'écoute de la matière ", Abbas Kiarostami, le cinéma à l'épreuve du réel, dir. Philippe Ragel, Yellow Now / LARA, 2008, p. 25.

\section{RÉSUMÉS}

Lorsqu'Abbas Kiarostami réalisait en 1987 Où est la maison de mon ami ?, il ne se doutait pas jusqu'où le désormais légendaire plan du chemin zébrant la colline le mènerait. L'étude présentée ici se propose d'observer l'évolution de ce motif itératif tout au long de l'œuvre qui suivrait. Comment, chemin faisant, son graphisme d'abord angulaire, saillant, bien accordé à la prise de risque et au régime d'indétermination de ce cinéma du réel, s'est peu à peu arrondi pour dessiner, à partir du Goût de la cerise, une ronde mélancolique qui préfigure un imaginaire calligraphique du paysage dont le photo-film Roads of Kiarostami apparaît comme le point d'acmé.

\section{AUTEUR}

\section{PHILIPPE RAGEL}

Maître de Conférences

Université de Toulouse II le Mirail 